\title{
Metered Dose Inhaler Dosage Form
}

National Cancer Institute

\section{Source}

National Cancer Institute. Metered Dose Inhaler Dosage Form. NCI Thesaurus. Code C91148.

A liquid medication delivered as a mist to be inhaled as a measured dose 